\title{
Fecal microbiota transplantation in alcohol related liver diseases
}

\author{
Saggere Muralikrishna Shasthry \\ Department of Hepatology, Institute of Liver and Biliary Sciences, New Delhi, India
}

The current standard of care for severe alcoholic hepatitis (SAH) has several limitations in that only up to one-third of patients are eligible for steroid therapy. Additionally, steroids have their own issues: a portion of patients do not respond, while there is doubtful long-term benefit in those who do and a large proportion are ineligible to receive steroids entirely and hence have no definitive options for treatment. As such, there is a large gap between the problem and the available solutions. Alcohol causes dysbiosis and also disrupts gut barrier function, consequently promoting the translocation of microbial lipopolysaccharide into the portal circulation and liver. Therefore, probiotics, prebiotics, antibiotics, or transplantation of gut microbiota are likely to attenuate the dysbiosis-related liver insult. Fecal microbiota transplantation (FMT) is expected to have a role in managing alcoholic liver disease in general and SAH in particular by correcting dysbiosis, the primary insult. Results from mouse studies have suggested beyond doubt that alcohol-related liver injury is transferrable and also treatable by adopting FMT from suitable donors. Initial human trials from our center have affirmed benefits in human subjects with SAH as well, with both improvements in disease severity and as well as the rate of survival. Further studies addressing the head-to-head comparison of steroids and FMT are ongoing. Available preliminary data are promising and FMT and/or gut microbial modulation might become the standard of care in the near future for managing alcohol-related liver diseases, especially alcoholic hepatitis, with greater applicability, improved acceptability, and minimal side effects. (Clin Mol Hepatol 2020;26:294-301)

Keywords: Fecal microbiota transplantation; Alcoholic liver diseases; Alcoholic hepatitis

\section{INTRODUCTION}

In recent years, we have seen increasing evidence regarding the role of gut microbiota in the pathogenesis and progression of many diseases. The liver, being the first line of filtration between the gut and the rest of the body, is exposed to the brunt of changes in the gut microbiome and hence is more likely to be affected by dysbiosis. It is likely that most liver diseases are responses of the liver to such changes in the gut microbiome. The gut mi- crobiome can be affected by the food we eat, drugs we take, and other aspects of our lifestyle including alcohol intake.

In correlation with changes in the lifestyle and improvements in the financial status, alcoholic liver disease has become the most common chronic liver disease, even in Asian countries. Nearly $1 \%$ of the global deaths and $50 \%$ of the cases of liver disease worldwide are caused by alcohol.' Alcoholic hepatitis, the most florid form of alcoholic liver disease, has a very high short-term mortality of up to $50 \%$ and no specific therapies are available other than Abbreviations:
FMT, fecal microbiota transplantation; MAIT, mucosa-associated invariant T; SAH,
severe alcoholic hepatitis

\author{
Corresponding author : Saggere Muralikrishna Shasthry \\ Department of Hepatology, Institute of Liver and Biliary Sciences, \\ Vasantkunj, New Delhi 110070, India \\ Tel: +91-11-46300000, Fax: +91-11-46300063 \\ E-mail: shasthry@gmail.com \\ https://orcid.org/0000-0002-5846-6263
}


steroids. Steroids also only show a limited utility in improving the short-term survival and boast no evidence of any long-term benefits. Additionally, only a small proportion of patients with alcoholic hepatitis are eligible to receive steroids. Thus, a large number of patients are either not eligible or do not respond to steroids and this group outnumbers those who do respond to steroids, leaving us without any specific therapeutic options for a majority of these individuals. ${ }^{2}$ Even liver transplantation is not feasible in most cases due to the presence of sepsis or recent alcohol consumption and many ethical and logistic issues are involved despite the documented safety and survival benefits of early liver transplantation in patients with severe alcoholic hepatitis $(\mathrm{SAH})$ not responding to medical management. ${ }^{3}$ Therefore, newer, more effective, and nontransplant therapeutic options for managing severe alcoholic hepatitis are needed.

Since gut dysbiosis, leaky gut, and products of the gut microbiome reaching the liver are the main culprits in the development of alcoholic hepatitis, ${ }^{4}$ targeting qualitative and quantitative changes in the gut microbiome remains an important strategy in developing new therapies for alcoholic hepatitis. ${ }^{5}$ Among others, the modulation of gut microbiota by fecal microbiota transplantation (FMT) has recently been conceptualized and evaluated as a potential therapeutic strategy in both preclinical and clinical studies.
The methods for evaluating the gut microbiota have also remarkably improved in recent years. Next-generation sequencing of bacterial DNA has helped in assessing the bacterial composition and community diversity from the phyla to the species level, without the need for cultures. ${ }^{6}$ Sequencing of the bacterial ribosomal RNA gene (16s rRNA) is widely available for assessing specific bacterial taxa and their relative abundances by referencing from online databases. ${ }^{7}$ Metagenomic analysis by the shotgun approach can assess all the genes present in a given sample and identify abundances of specific metabolic processes. ${ }^{8}$ In this review, we discuss the current status of FMT in managing alcoholic hepatitis and alcoholic liver disease.

\section{THE GUT MICROBIOME AND ITS ROLE IN THE PATHOGENESIS OF ALCOHOLIC LIVER DISEASE}

Prolonged alcohol consumption results in the development of fatty liver (due to increased fatty acid/triglyceride synthesis and as well as increased fatty acid influx), while additional unknown triggering events initiate steatohepatitis. Gut dysbiosis, greater gut permeability, and increased gut microbial products in the portal circulation are the most commonly reported triggers initiating al-

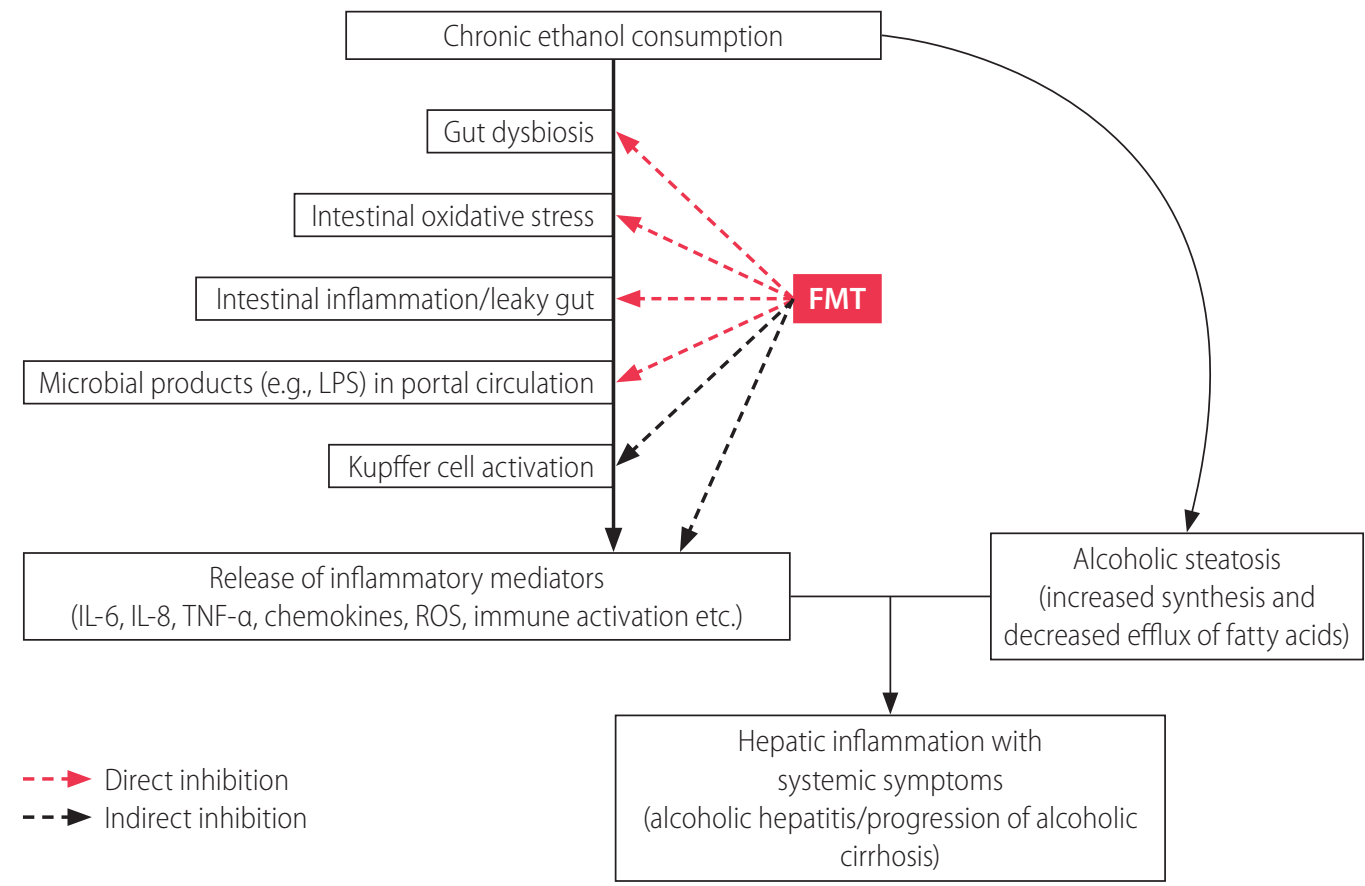

Figure 1. Role of the gut-liver axis in the pathogenesis of alcoholic hepatitis/alcoholic liver disease and potential mechanisms of action of FMT in the management of alcohol-associated liver diseases. LPS, lipopolysaccharide; IL-6, interleukin-6; IL-8, interleukin-8; TNF-a, tumor necrosis factor-alpha; ROS, reactive oxygen species; FMT, fecal microbiota transplantation. 
coholic hepatitis, ${ }^{2}$ prompting the activation of the innate and adaptive immune systems, hence the hepatic insult. Some questions like why only a small proportion of alcohol consumers develop liver disease and an even lesser proportion develop alcoholic hepatitis might have answers in the gut microbiome signatures they possess. Gut microbiome modulation may be an important and promising way of dealing with alcoholic liver disease (Fig. 1).

Normally, the gut microbiome boasts significant diversity, including bacteria, fungi, and viruses. Bacteria itself have multiple phylae, with many families, genera, and species. ${ }^{9}$ Given the presence of such a diverse population of organisms in the gut, with more than 150 times the active genes in the human body itself, and, together with its varied metabolites, the gut cannot just be a silent organ but rather holds an integral role in health and various diseases. Whenever the symbiotic relationship between the host and the gut microbiome is unbalanced (dysbiosis), the products from the gut contribute to the development of disease states, with alcoholic liver disease being one example.

Alcohol consumption causes disruption of the symbiosis between the gut microbiota and the host (dysbiosis), with additional disruption of the intestinal barrier, leading to the onset of leaky gut, ${ }_{10}^{10}$ as assessed by increased serum endotoxin levels in patients with alcoholic liver disease and alcoholic hepatitis." The causal role of gut microbial changes in the development of alcoholic liver disease has recently been assessed in many preclinical and clinical trials. Translocation of viable bacteria, bacterial metabolites, translocation of pattern-associated molecular patterns, intestinal inflammation, and changes in bile acids have been proposed as mechanisms of the onset and progression of alcohol-associated liver disease. ${ }^{12}$ Prolonged alcohol consumption is known to decrease the beneficial lactobacillus species irrespective of the presence of cirrhosis. ${ }^{13,14}$ Increases in proteobacteria and Fusobacteria and reductions in Lactobacillus and Bacteroidetes species are also well-established in alcoholic cirrhotics. ${ }^{15}$ Ethanol consumption has also been shown to be correlated with increases in the abundance of endotoxin-producing Enterobacteriaceae and a reduction in taxa that produce short-chain fatty acids such as Lachnospiraceae and Ruminococcaceae. ${ }^{16}$ An increase in the abundance of Candida species and decreased fungal diversity have also been observed in patients with alcoholic hepatitis. ${ }^{17}$ Alcohol consumption is shown to increase the bacterial colony counts (both aerobic as well as anaerobic), including more significantly in the proximal small bowel. ${ }^{18}$ In recent studies, it has been demonstrated that FMT from alcoholic hepatitis patients can produce necro-inflammatory changes in germ-free mice, proving the pathogenetic role of alco- hol-related gut dysbiosis in the development of liver injury. ${ }^{19}$

In addition to the occurrence of dysbiosis, changes in the fecal metabolites, such as reductions in short- and long-chain fatty acid levels and increases in the levels of both total and conjugated bile acids, are known to modulate the gut-liver axis in alcoholic liver disease. Disruption of the mucosal barrier function prompting changes in the mucous layer, reductions in secreted antimicrobial proteins like REG3G and mucin-2, the disruption of tight junctions leading to leaky gut, and alterations of the innate and adaptive immunity in the subepithelial space of the intestines have also been strongly linked to the pathogenesis of alcohol-induced liver disease. $^{12}$

\section{CURRENT STATUS OF GUT MICROBIOME MOD- ULATION IN ALCOHOL-ASSOCIATED LIVER DISEASES}

The gut microbiome is a very pliable environment that can be modulated with diet; pre-, pro- or antibiotics; and FMT. In preclinical trials, probiotics (Lactobacillus, Bifidobacterium) have been shown to improve alcohol-induced liver inflammation and gut leakiness. ${ }^{20,21}$ Prebiotics like fructo-oligosaccharides, which are substances that increase the number of beneficial gut microbes, improved alcohol-induced liver damage in mice. ${ }^{22}$ Interestingly, fecal microbiota manipulation by pectin, a fiber present in fruits, restored Bacteroides levels in mice and thus prevented liver injury by alcohol. ${ }^{23}$

Research suggests intestinal and circulating mucosa-associated invariant T (MAIT) cells are altered and involved in the increased number of bacterial infections present in patients with SAH. As such, FMT is likely to alter the MAIT cell dysfunction in $\mathrm{SAH}^{24} \mathrm{Al}-$ cohol-sensitive mice were found to have decreased Bateroidetes and increased Firmicutes and Actinobacteria populations relative to alcohol-resistant mice procured from the same laboratory. FMT from the alcohol-resistant mice to the alcohol-sensitive mice reversed the Bacteroidetes depletion and protected the mice from alcohol injury. ${ }^{23}$ In another translational study, Llopis et al. ${ }^{19}$ used germ-free humanized mice and completed FMT from alcoholic liver disease patients with and without SAH. With consequent alcohol feeding, the mice with FMT from SAH patients showed more severe liver inflammation, greater hepatic necrosis, higher intestinal permeability, and translocation of bacteria when compared with the mice which received FMT from non-SAH alcoholic patients. Also, the microbiome composition was distinctly different 
between the SAH and non-SAH alcoholic hepatitis patients as revealed by principal component analysis. Subsequently, a second FMT from patients without SAH into the mice who had earlier received FMT from SAH patients yielded improvements in liver lesions, confirming the etiopathological and therapeutic roles of the gut microbiome in severe alcoholic hepatitis. Current human data on FMT for alcohol-associated liver diseases are sparse; the available information along with the FMT preparation process will be discussed in detail in the subsequent sections.

\section{FMT IN ALCOHOLIC LIVER DISEASE}

Coprophagy (consumption of feces) is common in the animal kingdom. Transfaunation has been practiced for centuries in veterinary medicine for treating conditions like ruminal acidosis and chronic diarrhea. ${ }^{25}$ Transplanted fecal material from donors may possibly preserve thousands of functional bacterial species and eventually re-establish a healthy functional gut microbiome in the recipient. ${ }^{26}$ The concept of FMT is not new: traditional Chinese doctors in the fourth century AD described the use of FMT (orally) for treating patients with difficult diarrheas. ${ }^{27}$ The last decade has seen a resurgence in the utility of FMT in managing human diseases with the United States Food and Drug Administration approving FMT for managing difficult-to-treat Clostridium difficile infections. ${ }^{28}$

\section{FMT process}

\section{FMT donation and processing}

Donor screening is of the utmost importance and should identify a healthy donor without any active infections by conducting screenings for hepatitis B surface antigens, anti-hepatitis C antibodies, human immunodeficiency viruses 1 and 2 , and venereal disease research laboratory testing. The donor's stool should also test negative for ovarian cysts, Rotavirus antigens, Helicobacter pylori antigens, Cryptosporidium, Isospora (AFB stain), and C. difficile toxins. Donors should not have experienced recent alcohol intake (in the last 90 days), altered bowel movements, or recent antibiotic use (in the last 90 days) (Table 1). The donor should also be free of any significant co-morbidities or chronic ailments and preferably aged between 18 and 60 years. In general, a young, healthy, lean, individual, preferably a relative (who possibly would share the same kitchen/human leukocyte antigen alleles), is an ideal donor for FMT. A stool sample should be collect- ed in the early morning a clean plastic container, preferably at a place very near to the processing unit (the FMT laboratory) to be processed in an automated system for safe and early disposal of the filtrate containing the microbiome suspension without fibrous residue.

\section{Fecal preparation for FMT}

Fresh fecal samples are preferred over frozen or stored samples given safety, efficacy, and viability concerns. Some reports suggest the level of efficacy is equal between the stored samples and

Table 1. Donor screening for FMT

Donors were excluded if they had any one of the following
conditions or characteristics
Abnormal bowel motions
Obesity
Chronic alcohol intake
Active substance abuse or failed to provide consent
Age of less than 18 or more than 60 years
HBsAg, anti-HCV, HIV seropositivity
Gastroenteritis within the last 2 months
Inflammatory bowel disease
Current or past history of any malignancy
Diabetes, chronic kidney disease, coronary artery disease, stroke,
COPD

Antibiotic usage within 3 months at the time of enrolment

Elevated liver enzyme

Laboratory investigations for FMT donor screening
Complete blood count
Liver function testing
Fasting blood sugar
Renal function testing
Stool routine microscopy for ovarian cysts
Stool culture
H. pylori stool antigen testing
Stool modified ZN stain (Cryptospora and Isospora)
Clostridium difficile antigens and toxins
Rotavirus antigens
HBsAg, anti-HCV, HIV 1 and 2
VDRL

FMT, fecal microbiota transplantation; HBsAg, hepatitis B surface antigen; anti-HCV, anti-hepatitis C virus; HIV, human immunodeficiency virus; COPD, chronic obstructive pulmonary disease; VDRL, venereal disease research laboratory testing. 
the stored frozen samples ${ }^{29}$ but most prefer fresh samples due to concerns about the loss of a proportion of the viable microbiome. ${ }^{30-34}$ At many large-volume FMT centers, including ours, the FMT workflow (collection, transportation, preparation, and delivery) is designed to be completed within 3 hours and fresh stool samples are always preferred, although further research in this regard is warranted. Automated GenFMTer purification systems (FMT Medical, Nanjing, China) are also useful in facilitating clean handing of the samples and packaging of the fecal slurry in easily and safely transportable parcels. ${ }^{24-28,35,36}$

\section{Routes of administration}

\section{FMT route of administration}

As of today, the best route of administration is not confirmed from amongst the oral, nasogastric, nasoduodenal, nasojejunal, endoscopic, rectal, and colonoscopic options depending on the disease condition ${ }^{23,24}$ and there are no head-to-head comparisons available in the literature. However, in cirrhotics with dysbiosis predominantly involving the proximal small bowel, the upper gastrointestinal route of FMT administration seems more logical. At our center, we prefer adopting nasoduodenal tube placement for FMT instillation in our cirrhotics to avoid the risk of aspiration. ${ }^{29}$

\section{Current data for FMT in ALD}

We now know that the alcohol induces dysbiosis, including more so in the proximal small bowel, and preclinical studies have confirmed the role of gut microbiota modulation to prevent or improve liver injury by alcohol. However, the precise method of gut modulation and the optimal site, duration, and method of modulation are still less clear, with many human trials still in the preliminary stages. With the immense potential of modulation of the gut microbiota and its possible therapeutic implications, researchers to date have attempted FMT in patients with severe alcoholic hepatitis, hepatic encephalopathy, hepatitis B-related chronic liver diseases, and nonalcoholic fatty liver disease.

In one clinical pilot trial involving patients with $\mathrm{SAH}(\mathrm{n}=8)$, Phil-

Table 2. Current literature discussing modulation of the gut microbiome in the management of alcohol-associated liver diseases

\begin{tabular}{|c|c|c|c|}
\hline Study & Intervention & Trial details & Summary \\
\hline Kirpich et al. ${ }^{37}$ (2008) & $\begin{array}{l}\text { Bifidobacterium bifidum and } \\
\text { Lactobacillus plantarum 8PA3 } \\
\text { vs. standard therapy }\end{array}$ & $\begin{array}{l}\text { Randomized open-label trial } \\
\text { Hospitalized male patients with alcoholic } \\
\text { psychosis ( } \mathrm{n}=66 \text { total; } n=26 \text { had } \\
\text { alcoholic hepatitis) }\end{array}$ & $\begin{array}{l}\text { Reduced serum AST and ALT levels } \\
\text { and increased relative abundance of } \\
\text { Lactobacillus spp. and bifidobacteria } \\
\text { in patients receiving probiotics }\end{array}$ \\
\hline $\begin{array}{l}\text { Stadlbauer et al. }{ }^{38} \\
\text { (2008) }\end{array}$ & $\begin{array}{l}\text { Lactobacillus casei strain Shirota } \\
\text { three times daily (every } 8 \\
\text { hours) for } 4 \text { weeks }\end{array}$ & $\begin{array}{l}\text { Open-label study } \\
\text { Compensated alcoholic cirrhotics ( } n=10)\end{array}$ & $\begin{array}{l}\text { Neutrophilic phagocytic capacity } \\
\text { improved relative to baseline }\end{array}$ \\
\hline Han et al. ${ }^{39}$ (2015) & $\begin{array}{l}\text { Bacillus subtilis and } \\
\text { Enterococcus faecium vs. } \\
\text { placebo for } 7 \text { days }\end{array}$ & $\begin{array}{l}\text { Placebo-controlled trial } \\
\text { Admitted patients with alcoholic hepatitis } \\
(\mathrm{n}=117)\end{array}$ & $\begin{array}{l}\text { Improvements in liver function, } \\
\text { systemic inflammation, and } \\
\text { endotoxemia along with lower } \\
\text { colony-forming unit count of } \\
\text { Escherichia coli in the probiotic group }\end{array}$ \\
\hline Philips et al. ${ }^{35}$ (2017) & $\begin{array}{l}\text { FMT through nasoduodenal } \\
\text { tube for } 7 \text { days }\end{array}$ & $\begin{array}{l}\text { Open-label 1-year follow-up study } \\
\text { Steroid-ineligible male patients with } \\
\text { severe alcoholic hepatitis }(n=8)\end{array}$ & $\begin{array}{l}\text { Improved survival and liver function } \\
\text { in FMT group relative to among } \\
\text { historical controls } \\
\text { Reduction in the potentially pathogenic } \\
\text { species seen in the FMT group }\end{array}$ \\
\hline Philips et al. ${ }^{40}$ (2018) & $\begin{array}{l}\text { FMT daily for } 7 \text { days via } \\
\text { nasoduodenal tube vs. } \\
\text { corticosteroids, nutritional } \\
\text { therapy, or pentoxifylline }\end{array}$ & $\begin{array}{l}\text { Open-label study with 3-month follow-up } \\
\text { Alcoholic hepatitis patients (all males) } \\
\text { treated with FMT ( } n=16) \text {, pentoxifylline } \\
(n=10) \text {, corticosteroids }(n=8) \text {, nutritional } \\
\text { therapy }(n=17)\end{array}$ & $\begin{array}{l}\text { Three-month survival was highest in } \\
\text { the FMT group } \\
\text { Favorable gut microbial changes found } \\
\text { in the FMT group }\end{array}$ \\
\hline $\begin{array}{l}\text { Pande et al. (ongoing } \\
\text { trial; NCT03091010) }\end{array}$ & $\begin{array}{l}\text { FMT daily via nasoduodenal } \\
\text { tube for } 7 \text { days vs. } \\
\text { corticosteroids }\end{array}$ & $\begin{array}{l}\text { Randomized controlled trial assessing } \\
\text { 90-day survival between FMT and } \\
\text { corticosteroids }\end{array}$ & $\begin{array}{l}\text { Preliminary unpublished results } \\
\text { showing } 90 \text {-day survival benefits in } \\
\text { the FMT group }\end{array}$ \\
\hline
\end{tabular}

AST, aspartate transaminase; ALT, alanine transaminase; FMT, fecal microbiota transplantation. 
ips et al. ${ }^{35}$ administered FMT consecutively for seven days in steroid-ineligible patients and reported improvements at one year regarding survival in comparison with historical controls (87.5\% vs. 33.3\%). Both the relative abundance of Proteobacteria and a low abundance of Actinobacteria at baseline in SAH patients improved after FMT. Additionally, the coexistence of recipient and donor species was noted even at 6 to 12 months after FMT. This coexistence reiterates the fact that the donor bacteria modulate the preexisting recipient bacteria and suppresses their pathological nature by possibly increasing the biodiversity. In fact, changes have also been observed at the species level with a reduced abundance in the pathogenic species (Klebsiella pneumoniae, from 10\% to $1 \%$ by 1 year) and an increased relative abundance of beneficial species (Enterococcus villorum, Bifidobacterium longum, and Megasphaera elsdenii). At the metagenomic level, bile secretion, carotenoid synthesis, and pantothenate synthesis pathways, which were downregulated at baseline, improved during followup after FMT. This trial highlighted the safety of FMT even in the sickest group of SAH patients (i.e., steroid-ineligible patients) and further paved the way for future trials. Following this trial, a ran- domized controlled trial (NCT 03091010) involving the comparison patients with SAH receiving steroids or FMT from our center showed promising results, with an improved 90-day survival rate in the group receiving FMT relative to those given steroids revealed by the preliminary unpublished data $(n=112)$. Further detailed results of this trial will be of the utmost importance and may change the way we currently treat our patients with SAH (Table 2). ${ }^{35,37-40}$ To our knowledge, in the currently available literature, no major side effects attributed FMT have been described. Only abdominal distension due to gaseous bloating has been documented in a small proportion of patients.

Dysbiosis and the gut-liver axis are important and integral parts of the pathogenesis, progression, and outcomes of most liver diseases and even more so with respect to alcohol-associated liver diseases. Despite the immense potential perceived, current approaches in the modulation of the gut microbiome as therapies for liver disorders are inadequate. We must improve our protocols for FMT preparation, transport, and delivery so as to enhance both the quality (microbial diversity/richness) and shelf life of samples and alleviate aesthetic concerns to support greater con-

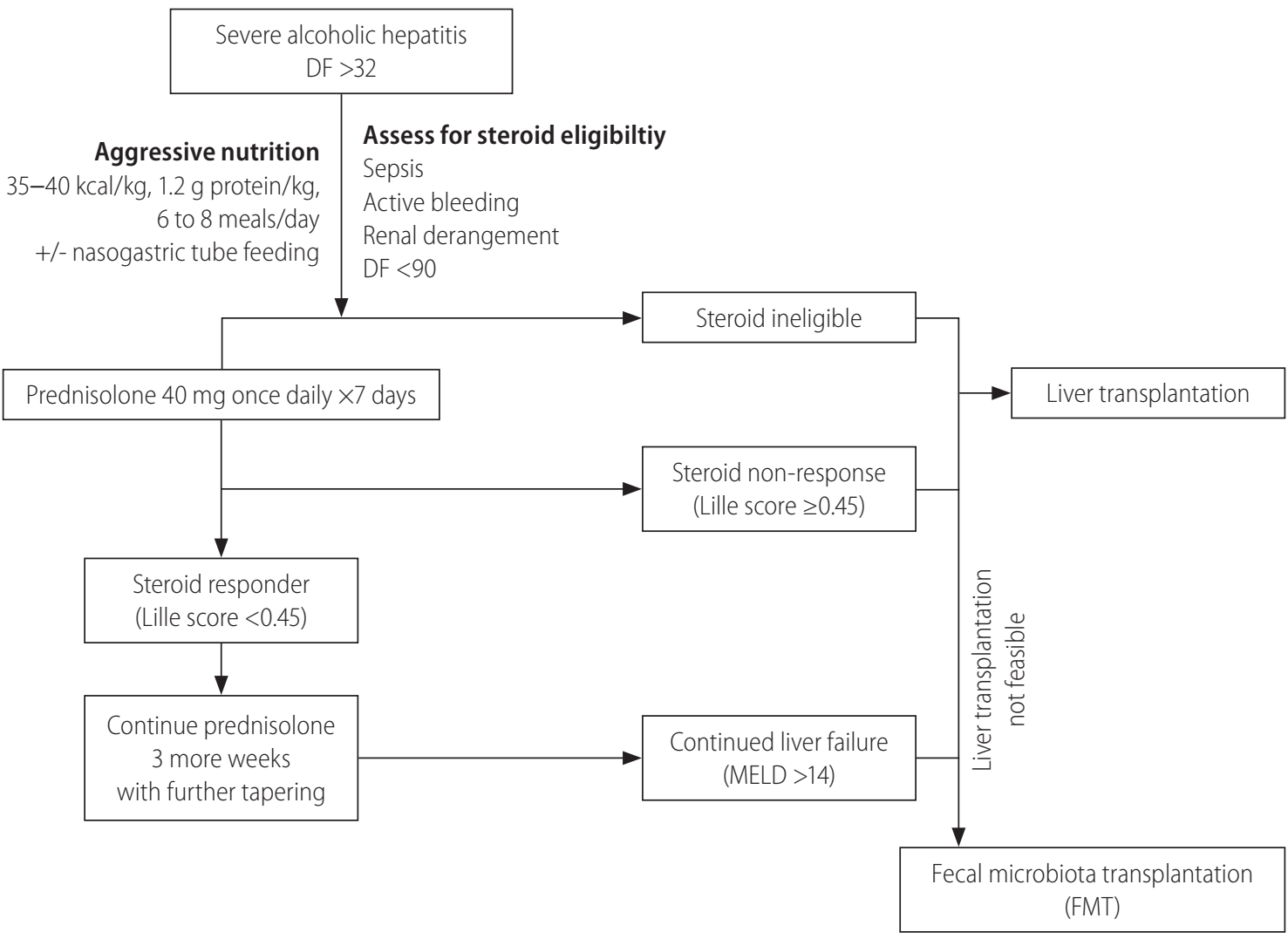

Figure 2. Current position for FMT in severe alcoholic hepatitis. DF, discriminant function; MELD, Model for End-stage Liver Disease. 
venience and improved efficacy. Also, the best route of administration according to the disease condition needs to be further explored—for example, colonic administration for difficult-to-treat C. difficile infections and proximal delivery for liver diseases including alcoholic liver disease where dysbiosis is more pronounced in the proximal bowel may be optimal techniques. The timing, quantity, frequency, and assessment of response to treatment all warrant continued exploration in future trials.

\section{CONCLUSION}

Current treatment options in the management of SAH remain primitive and rudimentary. Steroids are still the standard of care with doubtful long term benefits ${ }^{41}$ and only about one-third of patients of SAH are even eligible for steroids, of which only a proportion respond positively to therapy. A large number of patients with SAH are therefore either ineligible or nonresponsive. There is no blanket standard of care available to date as a standard of care to treat each and every patient with SAH that displays good long-term efficacy. In this regard, FMT stands a fair chance of attaining a position in the current SAH treatment algorithm, at least in steroid-ineligible or nonresponsive patients and in those with persistent liver failure (Model for End-stage Liver Disease score $>14$ points) despite the completion of steroids (Fig. 2). Further high-quality trials are required to address many unsolved issues.

\section{Conflicts of Interest}

The author has no conflicts to disclose.

\section{REFERENCES}

1. Gao B, Bataller R. Alcoholic liver disease: pathogenesis and new therapeutic targets. Gastroenterology 2011;141:1572-1585.

2. Shasthry SM, Sarin SK. New treatment options for alcoholic hepatitis. World J Gastroenterol 2016;22:3892-3906.

3. Mathurin P, Moreno C, Samuel D, Dumortier J, Salleron J, Durand F, et al. Early liver transplantation for severe alcoholic hepatitis. N Engl J Med 2011;365:1790-1800.

4. Lucey MR, Mathurin P, Morgan TR. Alcoholic hepatitis. N Engl J Med 2009;360:2758-2769.

5. Cassard AM, Ciocan D. Microbiota, a key player in alcoholic liver disease. Clin Mol Hepatol 2018;24:100-107.

6. Gillevet P, Sikaroodi M, Keshavarzian A, Mutlu EA. Quantitative assessment of the human gut microbiome using multitag pyrosequencing. Chem Biodivers 2010;7:1065-1075.

7. Lozupone CA, Stombaugh JI, Gordon JI, Jansson JK, Knight R. Diversity, stability and resilience of the human gut microbiota. Nature 2012:489:220-230.

8. Qin J, Li Y, Cai Z, Li S, Zhu J, Zhang F, et al. A metagenome-wide association study of gut microbiota in type 2 diabetes. Nature 2012;490:55-60.

9. Sender R, Fuchs S, Milo R. Are we really vastly outnumbered? Revisiting the ratio of bacterial to host cells in humans. Cell 2016;164:337-340.

10. Hartmann P, Chen P, Wang HJ, Wang L, McCole DF, Brandl K, et al. Deficiency of intestinal mucin-2 ameliorates experimental alcoholic liver disease in mice. Hepatology 2013;58:108-119.

11. Parlesak A, Schäfer C, Schütz T, Bode JC, Bode C. Increased intestinal permeability to macromolecules and endotoxemia in patients with chronic alcohol abuse in different stages of alcohol-induced liver disease. J Hepatol 2000;32:742-747.

12. Sarin SK, Pande A, Schnabl B. Microbiome as a therapeutic target in alcohol-related liver disease. J Hepatol 2019;70:260-272.

13. Bull-Otterson L, Feng W, Kirpich I, Wang Y, Qin X, Liu Y, et al. Metagenomic analyses of alcohol induced pathogenic alterations in the intestinal microbiome and the effect of Lactobacillus rhamnosus GG treatment. PLoS One 2013;8:e53028.

14. Leclercq S, Matamoros S, Cani PD, Neyrinck AM, Jamar F, Stärkel $P$, et al. Intestinal permeability, gut-bacterial dysbiosis, and behavioral markers of alcohol-dependence severity. Proc Natl Acad Sci U S A 2014;111:E4485-E4493.

15. Chen Y, Yang F, Lu H, Wang B, Chen Y, Lei D, et al. Characterization of fecal microbial communities in patients with liver cirrhosis. Hepatology 2011;54:562-572.

16. Bajaj JS, Kakiyama G, Zhao D, Takei H, Fagan A, Hylemon P, et al. Continued alcohol misuse in human cirrhosis is associated with an impaired gut-liver axis. Alcohol Clin Exp Res 2017;41:1857-1865.

17. Lang S, Duan Y, Liu J, Torralba MG, Kuelbs C, Ventura-Cots M, et al. Intestinal fungal dysbiosis and systemic immune response to fungi in patients with alcoholic hepatitis. Hepatology 2020;71:522-538.

18. Casafont Morencos F, de las Heras Castaño G, Martín Ramos L, López Arias MJ, Ledesma F, Pons Romero F. Small bowel bacterial overgrowth in patients with alcoholic cirrhosis. Dig Dis Sci 1996;41:552-556.

19. Llopis M, Cassard AM, Wrzosek L, Boschat L, Bruneau A, Ferrere G, et al. Intestinal microbiota contributes to individual susceptibility to alcoholic liver disease. Gut 2016;65:830-839.

20. Forsyth CB, Farhadi A, Jakate SM, Tang Y, Shaikh M, Keshavarzian A. Lactobacillus GG treatment ameliorates alcohol-induced intestinal oxidative stress, gut leakiness, and liver injury in a rat model of alcoholic steatohepatitis. Alcohol 2009;43:163-172. 
21. Chang B, Sang L, Wang Y, Tong J, Zhang D, Wang B. The protective effect of VSL\#3 on intestinal permeability in a rat model of alcoholic intestinal injury. BMC Gastroenterol 2013;13:151.

22. Yan AW, Fouts DE, Brandl J, Stärkel P, Torralba M, Schott E, et al. Enteric dysbiosis associated with a mouse model of alcoholic liver disease. Hepatology 2011;53:96-105.

23. Ferrere G, Wrzosek L, Cailleux F, Turpin W, Puchois V, Spatz $M$, et al. Fecal microbiota manipulation prevents dysbiosis and alcoholinduced liver injury in mice. J Hepatol 2017;66:806-815.

24. Gao B, Ma J, Xiang X. MAIT cells: a novel therapeutic target for alcoholic liver disease? Gut 2018;67:784-786.

25. DePeters EJ, George LW. Rumen transfaunation. Immunol Lett 2014;162(2 Pt A):69-76.

26. Qin J, Li R, Raes J, Arumugam M, Burgdorf KS, Manichanh C, et al. A human gut microbial gene catalogue established by metagenomic sequencing. Nature 2010;464:59-65.

27. Zhang F, Luo W, Shi Y, Fan Z, Ji G. Should we standardize the 1,700-year-old fecal microbiota transplantation? Am J Gastroenterol 2012;107:1755; author reply 1755-1756.

28. U.S. Food \& Drug Administration (FDA). Enforcement policy regarding investigational new drug requirements for use of fecal microbiota for transplantation to treat clostridium difficile infection not responsive to standard therapies. FDA web site, <https://www.fda. gov/regulatory-information/search-fda-guidance-documents/enforcement-policy-regarding-investigational-new-drug-requirementsuse-fecal-microbiota-0>. Accessed 29 Jan 2020.

29. Tang $G$, Yin W, Liu W. Is frozen fecal microbiota transplantation as effective as fresh fecal microbiota transplantation in patients with recurrent or refractory Clostridium difficile infection: a metaanalysis? Diagn Microbiol Infect Dis 2017;88:322-329.

30. Cui B, Feng $Q$, Wang $H$, Wang $M$, Peng Z, Li P, et al. Fecal microbiota transplantation through mid-gut for refractory Crohn's disease: safety, feasibility, and efficacy trial results. J Gastroenterol Hepatol 2015:30:51-58

31. Cui B, Li P, Xu L, Zhao Y, Wang H, Peng Z, et al. Step-up fecal microbiota transplantation strategy: a pilot study for steroid-dependent ulcerative colitis. J Transl Med 2015;13:298.
32. Zhang T, Xiang J, Cui B, He Z, Li P, Chen H, et al. Cost-effectiveness analysis of fecal microbiota transplantation for inflammatory bowel disease. Oncotarget 2017;8:88894-88903.

33. He Z, Cui BT, Zhang T, Li P, Long CY, Ji GZ, et al. Fecal microbiota transplantation cured epilepsy in a case with Crohn's disease: the first report. World J Gastroenterol 2017;23:3565-3568.

34. Uygun A, Ozturk K, Demirci H, Oger C, Avci IY, Turker T, et al. Fecal microbiota transplantation is a rescue treatment modality for refractory ulcerative colitis. Medicine (Baltimore) 2017;96:e6479.

35. Philips CA, Pande A, Shasthry SM, Jamwal KD, Khillan V, Chandel SS, et al. Healthy donor fecal microbiota transplantation in steroidineligible severe alcoholic hepatitis: a pilot study. Clin Gastroenterol Hepatol 2017;15:600-602.

36. He Z, Li P, Zhu J, Cui B, Xu L, Xiang J, et al. Multiple fresh fecal microbiota transplants induces and maintains clinical remission in Crohn's disease complicated with inflammatory mass. Sci Rep 2017;7:4753.

37. Kirpich IA, Solovieva NV, Leikhter SN, Shidakova NA, Lebedeva OV, Sidorov PI, et al. Probiotics restore bowel flora and improve liver enzymes in human alcohol-induced liver injury: a pilot study. Alcohol 2008;42:675-682.

38. Stadlbauer V, Mookerjee RP, Hodges S, Wright GA, Davies NA, Jalan R. Effect of probiotic treatment on deranged neutrophil function and cytokine responses in patients with compensated alcoholic cirrhosis. J Hepatol 2008;48:945-951.

39. Han SH, Suk KT, Kim DJ, Kim MY, Baik SK, Kim YD, et al. Effects of probiotics (cultured Lactobacillus subtilis/Streptococcus faecium) in the treatment of alcoholic hepatitis: randomized-controlled multicenter study. Eur I Gastroenterol Hepatol 2015;27:1300-1306.

40. Philips CA, Phadke N, Ganesan K, Ranade S, Augustine P. Corticosteroids, nutrition, pentoxifylline, or fecal microbiota transplantation for severe alcoholic hepatitis. Indian J Gastroenterol 2018;37:215225.

41. Thursz MR, Richardson $P$, Allison $M$, Austin A, Bowers $M$, Day $C P$, et al. Prednisolone or pentoxifylline for alcoholic hepatitis. N Engl J Med 2015:372:1619-1628. 